\title{
Diagnóstico y tratamiento de la tos crónica en pediatría
}

\author{
Diagnostic and treatment approach of chronic cough in childhood
}

\author{
Dr. Ricardo J. Saranz ${ }^{a}$, Dr. Alejandro Lozano ${ }^{a}$, Dra. Natalia A. Lozano ${ }^{a}$ y \\ Dr. José A. Castro Rodríguez
}

"La tos es el perro guardián de los pulmones que los protege de intrusos externos dañosos y de enemigos internos... No obstante, los médicos, a menudo, nos empeñamos en indicar fármacos que duermen al perro guardián cuando más lo necesitamos."

Chevallier JACKSON

Ther Gaz 1920;44:609-618

a. Servicio de Alergia e Inmunología, Clínica Universitaria Reina Fabiola. Cátedra de Inmunología, Facultad de Medicina,

Universidad

Católica de Córdoba, Argentina.

b. Unidad de

Neumonología

Pediátrica,

Departamento de

Pediatría y Medicina

Familiar, Escuela de

Medicina, Pontificia

Universidad Católica

de Chile, Santiago,

Chile.

Correspondencia:

Dr. Ricardo J. Saranz:

rsaranz@arnet.com.ar

Conflicto de intereses:

Ninguno que declarar.

Recibido: 11-10-2012

Aceptado: 28-12-2012

\section{RESUMEN}

La tos crónica es un síntoma de diversos procesos patológicos de origen respiratorio o extrarrespiratorio, con impacto negativo en la calidad de vida del niño y su familia. El pediatra debe guiar su acción en busca del diagnóstico etiológico. Una cuidadosa historia clínica es clave para el diagnóstico y una guía para solicitar los estudios complementarios necesarios para la detección de las causas definitivas. La exacta identificación etiológica de la tos crónica debe ser seguida de un tratamiento específico. El abordaje sintomático con antitusivos y mucolíticos es excepcionalmente necesario y debería reservarse para algunas situaciones de tos inespecífica sin causa aparente.

Palabras clave: tos crónica, infancia, diagnóstico, tratamiento.

\section{SUMMARY}

Chronic cough is a symptom of various respiratory and non-respiratory conditions with negative impact on quality of life of children and their families. The pediatricians should focus their efforts in search for etiological diagnosis. A careful medical history and physical examination are the mainstays of diagnosis and guidance to further studies that may contribute to detection of final causes.

The etiological identification of chronic cough must be followed by specific treatment. The symptomatic approach with antitussives and mucolytic drugs is exceptionally necessary and should be considered for some situations of nonspecific cough without any specific disease association.

Key words: chronic cough, childhood, diagnosis, treatment.

http:/ /dx.doi.org/10.5546/aap.2013.140

\section{DEFINICIÓN Y MAGNITUD DEL PROBLEMA}

La tos es un reflejo fisiológico complejo que consiste en una espiración violenta con el objetivo de liberar de secreciones, material extraño o broncoespasmo las vías aéreas de conducción, y proteger el sistema respiratorio de agentes químicos, infecciosos, mecánicos y térmicos. ${ }^{1,2}$ Puede transformarse en un síntoma perjudicial y molesto si es muy intensa o se prolonga en el tiempo, lo que impacta seriamente en la calidad de vida del niño y su familia, y genera un alto número de consultas médicas. ${ }^{3-5}$

Es de utilidad clínica definir la tos de acuerdo con su duración, su asociación con causas específicas y su respuesta terapéutica.

La tos crónica en pediatría se define como aquella que persiste por más de ocho semanas. ${ }^{6,7}$ No es una enfermedad per se, sino que suele ser la expresión clínica cardinal de numerosas patologías respiratorias y no respiratorias (tos específica). ${ }^{7-10}$ Cuando no puede reconocerse una causa se define como tos no específica, habitualmente de resolución espontánea.,11

Un $5 \%$ a $10 \%$ de la población pediátrica padece de tos crónica. ${ }^{3,12} \mathrm{Su}$ evaluación y diagnóstico requiere el adecuado conocimiento de las posibles causas. ${ }^{13-15}$ Es prioritaria la precisa identificación de la causa para la realización de un tratamiento etiológico. La tos refractaria, que persiste a pesar del tratamiento, no implica fortalecer un abordaje sintomático con antitusivos o expectorantes, que pueden tener efectos colaterales perjudiciales, ${ }^{16-18}$ si- 
no reforzar la estrategia diagnóstica en busca de la causa para indicar el tratamiento específico.

El objetivo de esta revisión es aportar las evidencias actuales acerca de la etiología, diagnóstico y terapéutica de la tos crónica en la niñez que le permitan al pediatra realizar un manejo adecuado de este molesto síntoma en su práctica diaria.

\section{ESPECTRO ETIOLÓGICO}

Numerosos factores pueden estimular el reflejo de la tos. Los más relevantes son hiperreactividad bronquial, infecciones, alergia, compresiones extrínsecas, alteraciones anatómicas, patologías de la vía aérea superior, macroaspiración y microaspiración respiratoria y causas psicógenas. ${ }^{1}$ En ocasiones pueden coexistir, lo que contribuye a una patogenia más compleja. 1,19,20

Las causas de la tos en los niños difieren de las de los adultos. ${ }^{21}$ Irwin y cols. ${ }^{19}$ demostraron, en una población mayoritariamente adulta, la descarga posnasal, el asma y el reflujo gastroesofágico (RGE) como las principales causas de tos crónica, que representan, en conjunto, el 86\% del total. En pediatría, la etiología de la tos puede variar según la edad. ${ }^{11,14}$ En los lactantes y preescolares las causas de tos crónica son diferentes de las de los escolares y adolescentes, que exhiben un espectro etiológico más parecido al de los adultos. ${ }^{10,14,22}$

La presencia de sintomatología en el primer año de vida sugiere anomalías congénitas, como fístula traqueoesofágica, anillo vascular, tronco braquiocefálico, trastornos neuromusculares y otras malformaciones de las vías aéreas. Las infecciones virales (p. ej., bronquiolitis) por Chlamydia, Bordetella pertussis, micobacterias (tuberculosis) y otras bacterias no deben soslayarse, al igual que la posibilidad de RGE, "tos como variante de asma" y fibrosis quística.

En la edad preescolar, se agrega el síndrome de tos asociado a patología de la vía aérea superior (UACS, del inglés upper airway cough syndrome ${ }^{23}$ (rinosinusitis, adenoiditis, infección e inflamación del anillo linfático de Waldeyer, etc.) y por efecto pasivo irritante del humo del cigarrillo como causas además de las mencionadas para menores de 2 años. La "tos como variante de asma" adquiere a esta edad más relevancia. La ocasionada por la aspiración de un cuerpo extraño, casi siempre paroxística, puede retrasar su comienzo y revestir la calidad de crónica.

La bronquitis bacteriana prolongada, recientemente descrita, es muy frecuente y se caracteriza por tos crónica productiva ocasionada por $\mathrm{Mo-}$ raxella catarrhalis, Haemophilus influenzae, Streptococcus pneumoniae; se resuelve con el uso de amoxicilina/ácido clavulánico o claritromicina durante 2 semanas. ${ }^{14,24,25}$

En el niño escolar y el adolescente, la rinosinusitis con descarga posnasal es la causa principal de tos; la "tos como variante de asma", exacerbada por el hábito de fumar $\mathrm{y}$, eventualmente la tos

Tabla 1. Historia clínica del niño con tos crónica: antecedentes

\begin{tabular}{|c|c|}
\hline Referencia de la historia clínica & Comentarios \\
\hline Edad de comienzo & Las causas de tos difieren según la edad \\
\hline Inicio neonatal & Puede sugerir anomalías congénitas respiratorias o cardíacas y trastornos neuromusculares \\
\hline Tiempo de evolución & Permite distinguir tos aguda, subaguda y crónica \\
\hline Calidad o tipo de tos & $\begin{array}{l}\text { Calificar la tos como metálica, perruna, staccatto, húmeda o productiva, seca, } \\
\text { espasmódica y paroxística es de guía diagnóstica }\end{array}$ \\
\hline Producción y calidad del esputo & $\begin{array}{l}\text { El esputo purulento es característico de bronquitis bacteriana, fibrosis quística, } \\
\text { bronquiectasia y síndrome de dismotilidad ciliar }\end{array}$ \\
\hline Ritmo horario & Tos nocturna: más frecuente en asma y rinosinusitis. Tos psicógena: se calma con el sueño \\
\hline Factores desencadenantes & Cambios de temperatura, frío, ejercicio, contaminantes y alérgenos principalmente del hogar \\
\hline Exposición al humo del cigarrillo & $\begin{array}{l}\text { Contaminante mórbido por excelencia; indagar sobre hábito de fumar de los padres o } \\
\text { cuidadores }\end{array}$ \\
\hline Existencia o no de síntomas asociados & Fundamental para diferenciar la tos "específica" de la "inespecífica" \\
\hline Respuesta a tratamientos efectuados & Determinar dosis, duración, grado de cumplimiento del tratamiento y respuesta obtenida \\
\hline $\begin{array}{l}\text { Enfermedad supurativa pulmonar, } \\
\text { infecciones atípicas }\end{array}$ & Alta posibilidad de inmunodeficiencia \\
\hline Neumonía recurrente & Probabilidad deinmunodeficiencia, anomalías congénitas del pulmón, fístula traqueoesofágica \\
\hline Uso de fármacos & Considerar inhibidores de la enzima convertidora de la angiotensina (IECA) \\
\hline
\end{tabular}


psicógena, que característicamente se calma durante el sueño, adquieren también relevancia., ${ }^{4,26}$

Una única causa es responsable de la tos crónica en la mayoría de los casos; no obstante, diferentes series que incluyeron niños y adultos hallaron dos causas responsables del síntoma en un $23 \%$ y $53 \%$ y hasta tres causas en un 3\% y $36 \% .^{19,20}$ Un reciente estudio en niños de 6 a 14 años detectó dos causas simultáneas en un $19,4 \%,{ }^{10}$ asociación no observada en otro estudio similar..$^{22}$ Esto tiene consecuencias terapéuticas directas: es indispensable el reconocimiento etiológico integral para acrecentar la posibilidad de éxito terapéutico.

Lo que conocemos:

- Las causas de tos crónica difieren entre la infancia y la edad adulta.

- En pediatría las causas varían según la edad.

- El origen de la tos puede incluir más de una causa.

Lo que falta conocer; perspectivas futuras:

- Qué factores pueden predecir la evolución natural de la tos crónica en los niños.

- Determinar la real incidencia de las causas mixtas de tos crónica en una población exclusivamente pediátrica.

\section{EVALUACIÓN DEL NIÑO CON TOS CRÓNICA \\ Historia clínica}

La historia clínica tiene una importancia diagnóstica fundamental. La anamnesis permite de- tectar y caracterizar el tiempo de comienzo y la evolución, el tipo de tos, el ritmo horario, los factores agravantes y desencadenantes, la producción y calidad del esputo, y los síntomas asociados $^{7,15,27}$ (Tabla 1).

La presencia de signos y síntomas orientadores de causas específicas de tos y la evaluación del crecimiento y desarrollo del niño deben valorarse mediante un detallado examen físico. ${ }^{8,27}$ (Tabla 2).

Debe considerarse la historia neonatal y alimentaria, el hábito de llevar objetos a la boca y los antecedentes personales y familiares de alergia. El pediatra debe revisar las vacunaciones realizadas, la presencia de irritantes y alérgenos hogareños, y las medicaciones prescritas. ${ }^{6}$

La tos asociada al asma es principalmente nocturna, espasmódica y se exacerba por el frío, los irritantes, los alérgenos y el ejercicio. ${ }^{3,28}$ Puede ser la única manifestación por mucho tiempo y retrasar el diagnóstico definitivo. El conocimiento de antecedentes personales y familiares de atopia puede ser orientador. ${ }^{3}$

La tos crónica asociada con expectoración purulenta sugiere bronquiectasias, enfermedad supurativa pulmonar o fibrosis quística. ${ }^{24} \mathrm{La}$ asociación con diarrea crónica, retraso del crecimiento, poliposis nasal o dedos "en palillo de tambor" es fuerte evidencia de fibrosis quística.

El niño con descarga posnasal manifiesta frecuentemente obstrucción nasal, halitosis y rinorrea mucopurulenta. ${ }^{23}$ La cefalea persistente puede ser un síntoma sugerente de sinusitis. ${ }^{29}$

TABLA 2. Historia clínica del niño con tos crónica: síntomas y signos

\begin{tabular}{ll}
\hline Síntoma/signo & Posible etiología \\
\hline Hallazgos auscultatorios pulmonares & Asma, bronquitis, cuerpo extraño, aspiración, anomalías congénitas, fibrosis quística \\
Soplo cardíaco & Enfermedad cardíaca \\
Dolor torácico & Asma, pleuritis \\
Deformidad torácica & Enfermedad pulmonar crónica grave \\
Tos productiva & Bronquitis crónica, enfermedad supurativa pulmonar, fibrosis quística \\
Dedos hipocráticos & Enfermedad supurativa pulmonar, fibrosis quística, cardiopatía \\
Disnea crónica & Enfermedad de la vía aérea o del parénquima pulmonar, enfermedad cardíaca \\
Disnea con ejercicio & Asma, enfermedad del parénquima pulmonar \\
Retraso del crecimiento & Enfermedad pulmonar o cardíaca grave, fibrosis quística \\
Tos antes o después de la alimentación & Reflujo gastroesofágico, aspiración primaria \\
Tos asociada a asfixia brusca & Aspiración de cuerpo extraño \\
Hemoptisis & Enfermedad supurativa pulmonar, anomalías vasculares, bronquitis \\
Fiebre & Tuberculosis,enfermedad supurativa pulmonar, bronquitisbacteriana, otrasinfecciones \\
Tos que calma de noche & Tos psicógena \\
\hline
\end{tabular}


El antecedente de síndrome febril recurrente con malestar general y tos generalmente productiva obliga a indagar acerca de los contactos y a sospechar una tuberculosis.

En los síndromes aspirativos la tos puede ligarse a regurgitación y ahogos, y exacerbarse durante o después de la alimentación. En ocasiones, se auscultan sibilancias y crepitantes basales. ${ }^{30}$

La tos psicógena es seca, de timbre perruno, repetitiva, frecuente durante el día y típicamente se calma con el sueño. Suele exacerbarse en presencia de padres o cuidadores, y disminuir ante actividades de distracción sociales o deportivas. Su diagnóstico es por exclusión en un niño sano y no mejora con antitusivos. ${ }^{26}$

\section{Estudios complementarios}

La necesidad de estudios complementarios costosos o riesgosos deberá estar avalada por una historia clínica que así lo sugiera. 11,13,15,27

La principal fortaleza de un estudio diagnóstico es descartar posibilidades diagnósticas sospechadas y la principal limitación de un resultado positivo es que no necesariamente debe ser considerado como regla diagnóstica. Es fundamental correlacionar el valor diagnóstico de una prueba con una respuesta terapéutica favorable para optimizar su utilidad. ${ }^{31}$

Una radiografía de tórax es el primer estudio para indicar; debe realizarse en casi todos los casos, permite valorar la presencia de una causa orgánica pulmonar de tos (Tabla 3) y es una guía para el algoritmo de estudios complementarios posteriores $^{6,8}$ (Figura 1). Si la radiografía de tórax es anormal (p. ej., presencia de lesiones localizadas o infiltrados difusos), existe la posibilidad de recurrir a técnicas más complejas de diagnóstico por imágenes, cultivo y citología de esputo y, finalmente, a la broncoscopia diagnóstica. ${ }^{7,8}$

En los niños mayores de 3 años, la espirometría puede detectar una obstrucción bronquial reversible, compatible con asma. ${ }^{3,7,28} \mathrm{Si}$ fuera normal con fuerte sospecha de asma, se podrían indicar exámenes más especializados, no siempre disponibles, como una prueba de provocación bronquial y estudios de inflamación de la vía aérea por medición de óxido nítrico exhalado o esputo inducido, debiéndose considerar la sensibilidad y especificidad del método empleado para optimizar su utilidad diagnóstica. ${ }^{6,32}$

La radiografía de senos paranasales tiene baja especificidad diagnóstica, que se mejora significativamente en combinación con los hallazgos clínicos para la determinación etiológica del UACS. ${ }^{29}$ La tomografía computarizada de senos paranasales no se recomienda rutinariamente para evaluar una sinusitis, si bien aporta mayor precisión diagnóstica. ${ }^{7,929}$

La fibroscopia rinofaringolaríngea permite detectar patologías orgánicas de la vía aérea superior (desvío del tabique nasal, hipertrofia de adenoides, pólipos), alteraciones en la deglución y signos indirectos de reflujo laringofaríngeo que pueden ser responsables de la tos..$^{29,30}$

El tránsito esofagogastroduodenal permite examinar cuerpos extraños en el esófago, fístulas traqueoesofágicas, compresiones exógenas del esófago y, secundariamente, RGE. Este último y

Tabla 3. Diagnóstico diferencial de causas específicas de tos crónica en pediatría

\begin{tabular}{|c|c|}
\hline Tos crónica en un niño aparentemente sano & Tos crónica en un niño con patología pulmonar \\
\hline $\begin{array}{l}\text { - } \text { Bronquitis recurrente } \\
\text { Viral } \\
\text { Bacteriana (Pertussis-Parapertussis) } \\
\text { (Chlamydia) } \\
\text { - Bronquitis bacteriana prolongada } \\
\text { - Rinosinusitis-descarga posnasal (UACS) } \\
\text { - Tos como variante de asma } \\
\text { - Reflujo gastroesofágico } \\
\text { - Tos psicógena } \\
\text { - Irritativa (fumador pasivo) }\end{array}$ & $\begin{array}{l}\text { - Fibrosis quística } \\
\text { - Inmunodeficiencias } \\
\text { - Discinesia ciliar primaria } \\
\text { - Aspiración pulmonar recurrente } \\
\text { - Aspiración de cuerpo extraño } \\
\text { - Infecciones } \\
\text { Mycoplasma } \\
\text { Tuberculosis } \\
\text { Neumonía bacteriana } \\
\text { - Anomalías congénitas } \\
\text { Fístula traqueoesofágica } \\
\text { Anillo vascular } \\
\text { Malformaciones de las vías aéreas } \\
\text { Trastornos neuromusculares }\end{array}$ \\
\hline
\end{tabular}


el reflujo laringofaríngeo deben sospecharse en todos los casos de tos de causa indeterminada. ${ }^{30}$ Aquí puede ser útil el monitoreo del pH esofágico de 24 horas, aunque un estudio normal no descarta el reflujo no ácido como causa de tos asociada, que puede ser detectado por impedanciometría. ${ }^{6,33}$

La valoración cuantitativa y funcional de las inmunoglobulinas deberá considerarse en los niños con tos persistente secundaria a infecciones bacterianas recurrentes. ${ }^{7}$ El diagnóstico de alergia con pruebas cutáneas con alérgenos o técnicas in vitro se reservará al especialista. ${ }^{6}$
El estudio de sensibilidad tuberculínica (PPD) es imprescindible si se detectan antecedentes epidemiológicos de contactos con tuberculosis, incluido todo el grupo familiar si fuera necesario.

Lo que conocemos:

- Una exhaustiva historia clínica y el examen físico son los pilares diagnósticos fundamentales y guía para la solicitud de estudios complementarios.

- La comprobación de una causa no necesariamente significa que esta sea responsable de la tos.

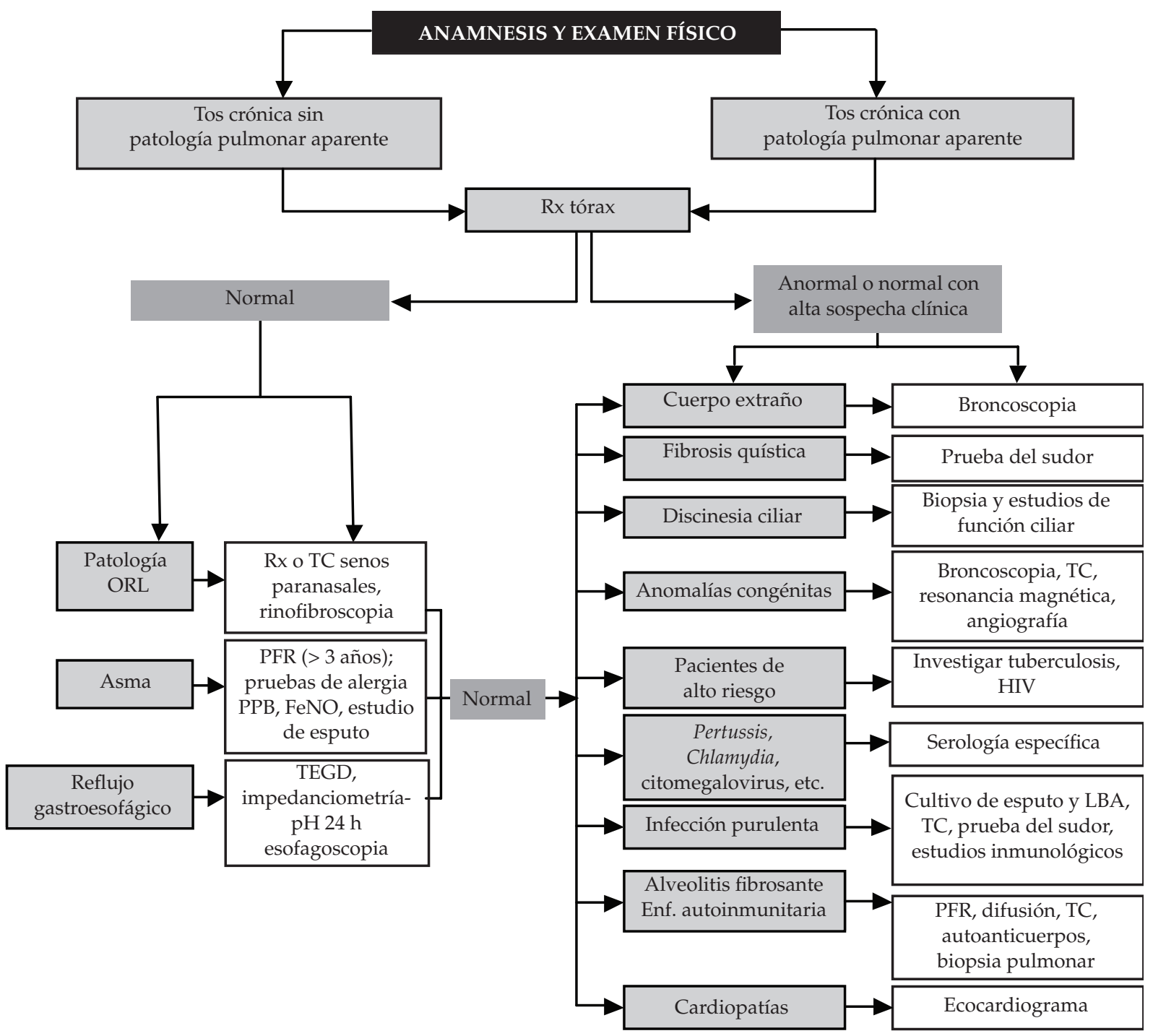

Rx: radiografía; TC: tomografía computarizada; PFR: prueba funcional respiratoria; PPB: prueba de provocación bronquial; FeNO: óxido nítrico exhalado; TEGD: tránsito esofagogastroduodenal; $\mathrm{pH} 24$ h: $\mathrm{pH}$-metría de 24 horas; LBA: lavado broncoalveolar. 
- Una prueba diagnóstica en tos crónica solo tiene consistencia si existe resolución sintomática con la terapéutica específica.

Lo que falta conocer; perspectivas futuras:

- Determinar la sensibilidad, especificidad, valor predictivo positivo, valor predictivo negativo, coeficiente de probabilidad positivo y negativo de los diferentes métodos diagnósticos de las causas de tos crónica en pediatría.

\section{TRATAMIENTO}

Antes de indicar cualquier medicación sintomática, el pediatra debería realizar una exhaustiva investigación causal. El análisis de la evidencia según el sistema GRADE (The Grading of Recommendations Assessment, Development and Evaluation) establece fortalezas de recomendaciones terapéuticas más rigurosas. . $^{11,34,35}$

Los objetivos del tratamiento de la tos crónica consisten primariamente en eliminar el agente causal, promover la broncodilatación, movilizar las secreciones facilitando la expectoración y, en última instancia, suprimir la estimulación de receptores periféricos y deprimir el centro de la tos.

\section{Tratamiento de la tos crónica con causa específica}

a. La tos crónica debida a asma requiere tratamiento broncodilatador con agonistas $\beta_{2}$ a demanda. El uso de esteroides por vía inhalatoria puede ser necesario, al menos por 4 a 8 semanas, para definir su efecto final..$^{6,28}$ Se sugieren medidas de evitación de alérgenos e irritantes, como la exposición al humo de cigarrillo (fortaleza de recomendación GRADE: fuerte). ${ }^{11}$

b. Tos asociada a rinitis alérgica: evitación de alérgenos, antihistamínicos y esteroides nasales (fortaleza de recomendación GRADE: débil). ${ }^{11}$ Aun así, en un niño con tos, la patología de la vía aérea superior no debiera soslayarse, y si existiera sinusitis bacteriana, es imperativa la terapia con antibióticos. . $3,29^{29}$

c. En la tos causada por reflujo gastroesofágico el ensayo empírico con inhibidores de la bomba de protones por 8 a 12 semanas no tiene recomendación específica de GRADE en los niños, ${ }^{11,36}$ al igual que la fundoplicatura laparoscópica. ${ }^{11}$

d. En la tos de causa infecciosa, el tratamiento es específico según la causa. La producida por bronquitis bacteriana prolongada requiere amoxicilina-ácido clavulánico o claritromicina por 2 a 6 semanas (recomendación GRA-
DE: fuerte). ${ }^{11,14,25}$ La terapia antituberculosa debe indicarse con diagnóstico confirmado de tuberculosis. Las causas virales más comunes de infección respiratoria generalmente son autolimitadas. $^{6}$

e. La tos psicogénica necesita la exploración de las circunstancias de estrés que envuelven el ámbito familiar y escolar. Puede requerir abordaje psicológico (fortaleza de recomendación GRADE: fuerte) ${ }^{6,7,11,26}$

f. Para cualquier tipo de tos la cesación del hábito de fumar de los padres tiene la mayor fortaleza de recomendación según el sistema GRADE. ${ }^{6,7,11}$

\section{Tos crónica inespecífica:}

\section{utilidad del tratamiento sintomático}

La conducta de "observar, esperar y revisar" tiene recomendación fuerte para evitar una medicación innecesaria cuyo efecto, generalmente, no supera al placebo ${ }^{37}$ y porque, en la mayoría de los casos, la tos inespecífica tiene resolución espontánea. $7,11,14$

En determinadas ocasiones, la tos produce efectos perjudiciales, como dolor torácico, fatiga, vómitos, cefaleas o trastornos del sueño, por lo que puede ser necesario un tratamiento sintomático.

Un ensayo empírico con esteroides inhalatorios y antihistamínicos en la tos sin causa aparente no tiene recomendación basada en la evidencia. ${ }^{6,11}$ Con el uso de cualquier medicación sintomática es conveniente una revaluación a las 2 a 3 semanas, tiempo en que es esperable la respuesta terapéutica (fortaleza de recomendación GRADE: moderada). ${ }^{11}$

Existe una amplia variedad de antitusivos clasificados según el sitio de acción. Los agentes narcóticos y no narcóticos de acción central actúan deprimiendo el centro integrador del reflejo, mientras que los de acción periférica deprimen los receptores donde se origina el reflejo tusígeno.

De los antitusivos de acción central la codeína es uno de los más potentes supresores de la tos, pero por ser narcótico, debe usarse el menor tiempo posible porque puede causar adicción. ${ }^{16}$ Además, puede producir sedación, mareos, intolerancia digestiva y sequedad bucal. ${ }^{17}$

Los derivados sintéticos de la codeína y la morfina, como oxicodona e hidrocodona, tienen alta eficacia, pero pueden causar los mismos efectos colaterales que sus drogas madres. Existe, según GRADE, una fuerte recomendación en contra de su uso. ${ }^{11}$ 
De los antitusivos no narcóticos, el dextrometorfano es equipotente con la codeína, actuando a nivel central. ${ }^{16,38}$ Es mejor tolerado pero, en ocasiones, tiene efectos gastrointestinales indeseables, aunque en las dosis recomendadas no produce sedación ni la depresión respiratoria que ocasiona la codeína y rara vez genera adicción. ${ }^{18}$ Noscapina, clofedianol, clobutinol, oxeladina y butamirato completan la lista de antitusivos no narcóticos aconsejables preferentemente para niños mayores de 6 años. ${ }^{38}$

Los expectorantes, como guaifenesina, estimulan la producción de secreciones del tracto respiratorio y se presentan muchas veces combinados con antitusivos. La bromhexina y el ambroxol, disponibles en nuestra farmacopea, pueden producir náuseas o vómitos por irritación gástrica. ${ }^{38}$

De los mucolíticos, la N-acetilcisteína interfiere las uniones disulfuro en el moco, disminuye marcadamente la viscosidad de este y tiene algún efecto antioxidante. ${ }^{39}$ No obstante, su utilidad en la tos crónica no está bien definida. Reducir el uso de mucolíticos y expectorantes tiene una fuerte recomendación GRADE. ${ }^{11}$

A pesar de estas advertencias, las medicaciones sintomáticas contra la tos siguen siendo, como hace casi un siglo, las más frecuentemente recetadas por el médico y consumidas por los pacientes. ${ }^{21,40,41}$

Lo que conocemos:

- El tratamiento de la tos crónica es más eficaz cuando se identifica y aborda la causa precisa.

- La terapia con antitusivos y mucolíticos puede contribuir al alivio del síntoma cuando la tos no sirve para fines fisiológicos como ocurre, por ejemplo, en la tos irritativa, seca y prolongada posviral, o para evitar eventuales complicaciones.

- La tos productiva no debería suprimirse, puesto que la retención de secreciones puede llevar a una prolongación del síntoma y de su enfermedad causal.

- Muchas combinaciones terapéuticas (p. ej., antitusivos y mucolíticos) carecen de sentido, ya que sus componentes tienen efectos contrapuestos y posibles efectos colaterales, tóxicos $\mathrm{o}$ adictivos.

Lo que falta conocer; perspectivas futuras:

- Promover una mayor concientización entre los pediatras sobre un uso más racional y adecuado de las medicaciones sintomáticas.

- Desarrollar nuevos antitusivos para disminuir la sensibilidad del reflejo de la tos.
- Efectuar estudios doble ciego controlados con placebo sobre antitusivos y establecer la real eficacia del tratamiento de algunas de las causas específicas de la tos en pediatría.

- Existen algunos protocolos promisorios en plena ejecución que permitirán diseñar algoritmos de manejo diagnóstico-terapéutico de la tos crónica en pediatría para usar en la consulta ambulatoria.

\section{CONCLUSIONES}

La tos es un síntoma cardinal de numerosas enfermedades respiratorias y no respiratorias. Su calidad de crónica requiere una evaluación diagnóstica ordenada y precisa. En los niños el espectro etiológico es diferente al del adulto, con consecuentes implicaciones terapéuticas.

La identificación causal debe ser seguida de un tratamiento específico. La terapéutica sintomática rara vez es necesaria, no está exenta de efectos colaterales indeseables y solo debe considerarse como adyuvante en caso de tos "irritativa" sin causa demostrable o como complemento del tratamiento específico.

\section{BIBLIOGRAFÍA}

1. Mc Cool DF, Leith DE. Pathophysiology of cough. Clin Chest Med 1987;8:189-95.

2. Canning BJ. Anatomy and neurophysiology of the cough reflex: ACCP evidence-based clinical Practice Guidelines. Chest 2006;129:33-47.

3. Faniran AO, Peat JK, Woolcock AJ. Persistent cough: is it asthma? Arch Dis Child 1998; 79:411-14.

4. RankMA, Kelkar P, Oppenheimer JJ. Taming chronic cough. Ann Allergy Asthma Immunol 2007;98:305-13.

5. Marchant JM, Newcombe PA, Juniper EF, Sheffield JK, et al. What is the burden of chronic cough for families? Chest 2008;134:303-9.

6. Shields MD, Bush A, Everard M L, McKenzie S, Primhak $\mathrm{R}$, etal. Recommendations for the assessment and management of cough in children. Thorax 2008;63(Suppl. III):iii1iii15.

7. Goldsobel AB, Chipps BE. Cough in the pediatric population. J Pediatr 2010:156:352-8.

8. de Jongste JC, Shields MD. Cough 2: Chronic cough in children. Thorax 2003;58:998-1003.

9. Chang AB, Glomb WB. Guidelines for evaluating chronic cough in pediatrics: ACCP Evidence-Based Clinical Practice Guidelines. Chest 2006;129:260-83.

10. Asiloy S, Bayram E, Agin H, Apa H, Can D, et al. Evaluation of chronic cough in children. Chest 2008;134:1122-8.

11. Gibson PG, Chang AB, Glasgow NJ, Holmes PW, et al. CICADA: Cough in Children and Adults: Diagnosis and Assessment. Australian Cough Guidelines summary statement. MJA 2010;192:265-71.

12. Chang AB, Berkowitz RG. Cough in the pediatric population. Otolaryngol Clin N Am 2010;43:181-98.

13. Marchant JM, Masters IB, Taylor SM, Chang AB. Utility of signs and symptoms of chronic cough in predicting specific cause in children. Thorax 2006;61:694-8. 
14. Marchant JM, Masters IB, Taylor SM, Cox NC, et al. Evaluation and outcome of young children with chronic cough. Chest 2006;129:1132-41.

15. Massie J. Cough in children: when does it matter? Ped Respir Rev 2006;7:9-14.

16. American Academy of Pediatrics Committee on Drugs. Use of codeine and dextromethorphan-containing cough remedies in children. Pediatrics 1997;99:918-20.

17. Schroeder K, Fahey T. Over-the-counter medications for acute cough in children and adults in ambulatory settings. Cochrane Database Syst Rev 2008;1:CD001831.

18. Schaefer MK, Shehab N, Cohen AL, Budnitz DS. Adverse events from cough and cold medications in children. $P e^{-}$ diatrics 2008;121:783-7.

19. Irwin RS, Curley FJ, French CL. Chronic cough: The spectrum and frequency of causes, key components of the diagnostic evaluation and outcome of specific therapy. Am Rev Respir Dis 1990;141:640-7.

20. French CL, Irwin RS, Curley FJ, Krikorian CJ. Impact of chronic cough on quality of life. Arch Intern Med 1998;158:1657-61.

21. Chang AB. Cough: are children really different to adults? Cough 2005;1:7-15.

22. Khoshoo V, Edell D, Mohnot S, Haydel R, et al. Associated factors in children with chronic cough. Chest 2009;136: 811-5.

23. Lack G. Pediatric allergic rhinitis and comorbid disorders. J Allergy Clin Immunol 2001;108:S9-15.

24. Chang AB, Redding GJ, Everard ML. Chronic wet cough: protracted bronchitis, chronic suppurative lung disease and bronchiectasis. Pediatr Pulmonol 2008;43:519-31.

25. Marchant J, Masters IB, Champion A, Petsky H, Chang AB. Randomised controlled trial of amoxycillin clavulanate in children with chronic wet cough. Thorax 2012;67:689-93.

26. Ramanuja S, Kelkar P. Habit cough. Ann Allergy Asthma Immunol 2009;102:91-5.

27. Brodlie M, Graham C, McKean MC. Childhood cough. BMJ 2012;344:e1177.

28. Niimi A. Cough variant asthma: A major cause of chronic cough. Clin Pulm Med 2008;15:189-96.

29. Pratter MR. Chronic upper airway cough syndrome secondary to rhinosinus diseases (previously referred to as postnasal drip syndrome). ACCP evidence-based clinical practice guidelines. Chest 2006;129:63S-71S.

30. Irwin RS. Chronic cough due to gastroesophageal reflux disease ACCP evidence-based clinical practice guidelines. Chest 2006;129:80S-94S.

31. Irwin RS. Introduction to the diagnosis and management of cough. ACCP evidence-based clinical practice guidelines. Chest 2006;129:25S-27S.

32. van Asperen PP. Cough and asthma. Pediatr Respir Rev 2006;7:26-30.

33. El-Sayed Ali M. Laryngopharyngeal reflux: diagnosis and treatment of a controversial disease. Curr Opin Allergy Clin Immunol 2008;8:28-33.

34. Jaeschke R, Guyatt GH, Dellinger P, et al. Use of GRADE grid to reach decisions on clinical practice guidelines when consensus is elusive. BMJ 2008;337:a744.

35. Terracciano L, Brozekb J, Compalati E, Schünemann H. GRADE system: new paradigm. Curr Opin Allergy Clin Immunol 2010,10:377-83.

36. Chang AB, Lasserson TJ, Kiljander TO, Connor FL, Gaffney JT, Garske LA. Systematic review and meta-analysis of randomized controlled trials of gastro-oesophageal reflux interventions for chronic cough associated with gastro-oesophageal reflux. BMJ 2006;332:11-7.

37. Paul IM. Therapeutic options for acute cough due to upper respiratory infections in children. Lung 2012;190:41-4.

38. Taylor JA, Novack AH, Almquist JR, Rogers JE. Efficacy of cough suppresants in children. J Pediatr 1993;122:799-802.

39. Kelly HW. Mucolytic therapy: what, when, where, why, and what is the evidence? Pediatr Allergy Immunol Pulmonol 2010;23:151-4.

40. Jackson C. Postulates on cough reflex in some of its medical and surgical phases. Ther Gaz 1920;44:609-18.

41. Cano Garcinuño A, Casares Alonso I, Rodríguez Barbero J, Pérez García I, Blanco Quirós A. Prescripción de fármacos anticatarrales de uso sistémico a niños de 0-13 años. Un problema no resuelto. An Pediatr (Barc) 2012 (en prensa). [Consulta: 24 de agosto de 2012]. Disponible en: http:// apps.elsevier.es / watermark/ctl_servlet?_f=10\&pident_ articulo $=15199 \&$ pident_usuario $=0 \&$ pcontactid $=\&$ pident revista $=37 \& t y=151$ \&accion $=$ L\&origen $=$ elsevier\&we $b=\mathrm{w}$ ww.elsevier.es\&lan=es\&fichero=S1695-4033(12)00187-7 . pdf\&eop=1. 